\title{
Regional Brain Activation During Meditation Shows Time and Practice Effects: An Exploratory FMRI Study ${ }^{\dagger}$
}

\author{
E. Baron Short ${ }^{1,2}$, Samet Kose ${ }^{1,2}$, Qiwen Mu ${ }^{1}$, Jeffery Borckardt ${ }^{1}$, Andrew Newberg ${ }^{3}$, \\ Mark S. George ${ }^{1,2,4}$ and F. Andrew Kozel ${ }^{1,2,4,5}$
}

${ }^{1}$ Brain Stimulation Laboratory, Psychiatry, Medical University of South Carolina, ${ }^{2}$ Ralph H. Johnson VA Medical Center, Charleston, SC, ${ }^{3}$ Radiology, Division of Nuclear Medicine, University of Pennsylvania Medical Center, Philadelphia, PA, ${ }^{4}$ Center for Advanced Imaging Research, Medical University of South Carolina, Charleston, SC and ${ }^{5}$ Psychiatry, University of Texas Southwestern Medical Center, Dallas, TX, USA.

\begin{abstract}
Meditation involves attentional regulation and may lead to increased activity in brain regions associated with attention such as dorsal lateral prefrontal cortex (DLPFC) and anterior cingulate cortex (ACC). Using functional magnetic resonance imaging, we examined whether DLPFC and ACC were activated during meditation. Subjects who meditate were recruited and scanned on a 3.0 Tesla scanner. Subjects meditated for four sessions of $12 \mathrm{~min}$ and performed four sessions of a $6 \mathrm{~min}$ control task. Individual and group t-maps were generated of overall meditation response versus control response and late meditation response versus early meditation response for each subject and time courses were plotted. For the overall group $(n=13)$, and using an overall brain analysis, there were no statistically significant regional activations of interest using conservative thresholds. A region of interest analysis of the entire group time courses of DLPFC and ACC were statistically more active throughout meditation in comparison to the control task. Moreover, dividing the cohort into short $(n=8)$ and long-term $(n=5)$ practitioners $(>10$ years) revealed that the time courses of long-term practitioners had significantly more consistent and sustained activation in the DLPFC and the ACC during meditation versus control in comparison to short-term practitioners. The regional brain activations in the more practised subjects may correlate with better sustained attention and attentional error monitoring. In summary, brain regions associated with attention vary over the time of a meditation session and may differ between long- and short-term meditation practitioners.
\end{abstract}

Keywords: anterior cingulate cortex - attention-dorsal lateral prefrontal cortex-functional MRI-meditation - time courses

For reprints and all correspondence: Baron Short, MD; Brain Stimulation Laboratory; Institute of Psychiatry, 67 President Street, 3North; Charleston, SC 29425, USA. Tel: 1(843) 792-0199;

Fax: 1(843)792-0159; E-mail: shorteb@musc.edu or shorteb@gmail.com

'Portions of this work were presented at the annual meeting of the Society of Biological Psychiatry, 2005, Atlanta, GA and Career Development Institute, 2006, Pittsburgh, PA, The Mind and Life Summer Research Institute, 2006, Garrison, NY and American Psychiatric Association, 2007, San Diego, CA, USA.

\section{Introduction}

Meditation involves attentional, emotional, cognitive and perceptual changes involving multiple brain regions (1). Despite multiple brain regions of activation during different types of meditation, frontal/prefrontal regions are most frequently activated and may be related to increased attentional requirements of meditation tasks (2). Attentional regulation is a common cognitive function associated with divergent meditation methods (2). The dorsal lateral prefrontal cortex (DLPFC) and anterior 
cingulate cortex (ACC) are involved in attentional control $(3,4)$, selective attention (5), attention shifting (6), monitoring $(7,8)$ and response selection (7-9). Thus DLPFC and ACC, as neural components of an attentional control circuit, may be activated during meditation to selectively attend to an object of concentration, monitor when attention has wandered elsewhere and redirect attention back to the object of concentration.

Three recent publications suggest neuroanatomical and attentional alterations associated with long-term meditation practice. Twenty subjects with long-term Insight meditation practice in comparison with matched controls had thicker prefrontal and insular cortex; regions which are associated with attention, interoception and sensory processing (10). In a study with 23 long-term Tibetan Buddhist monks, changes in binocular rivalry were measured after concentrative meditation techniques. Binocular rivalry occurs with an ambiguous visual data set that may be perceived as more than one type of image but perceptually constructed as one image at a time. It is used by neuroscientists to measure the alteration of attention fluctuations in conscious awareness despite constant external stimulation. Half of the subjects showed markedly reduced binocular rivalry after engaging in Tibetan one-pointed techniques, while novice practitioners showed no difference (11). In a third study, 13 age-matched Zen practitioners were compared with controls. While controls had a negative correlation of both gray matter volume and attentional performance with age, this was not found in the Zen practitioners (12). These studies suggest that some forms of long-term meditation practice may enhance attentional control and corresponding neurological structures (13). These changes were not reported in shorter term practitioners and may indicate a difference in the attentional systems between short- and long-term practitioners.

In order to extend the literature and test several prior studies for replication, we hypothesized that brain regions associated with executive attention would be more activated during meditation versus simple non-meditative tasks. Specifically, there would be increased activity in the DLPFC and ACC during meditation versus control activity. We additionally sought to examine changes in brain activity over time during the meditation sessions, reasoning that regional brain activation might be more prominent later in a single meditation session. Finally, we tested whether brain activity differed between long-term and short-term practitioners during meditation.

\section{Subjects and Methods}

\section{Subjects}

Right-handed subjects aged 18-60 years with at least 4 years of regular meditative practices from any meditative tradition were recruited. After providing written informed consent approved by the MUSC Office of Research Integrity, subjects were evaluated and included only if they routinely engaged in breath awareness meditation. Subjects were excluded who had a current or past DSM-IV Axis I disorder, a history of CNS disease, currently unstable medical conditions, current psychotropic medication, caffeine abuse, nicotine use, pregnant or lactating females or any metal implants. For meditation in the scanner, subjects were asked to keep their eyes open and to focus and only observe inhalation and exhalation. During the control period, subjects viewed a series of geometric images every $6 \mathrm{~s}$ and were asked to determine whether they were blue or yellow and select the appropriate button on a functional imaging glove system. The control task was chosen to be dissimilar from a resting condition because we were concerned that subjects might inadvertently meditate in a resting control condition. Subjects completed two runs that were (Run1 and Run2) randomized using a coin flip by order of whether the control or meditation task was first during the fMRI session. Each run consisted of two $12 \mathrm{~min}$. meditation and two $6 \mathrm{~min}$. control blocks.

\section{Functional MRI Data Acquisition}

All imaging was performed using a Philips Intera 3.0 Tesla MRI scanner and a multi-channel acquisition (SENSE) head coil. A high-resolution 142-slice $1 \mathrm{~mm}$ thick sagittal T1-weighted structural scan was acquired. For both runs, 541 echoplanar imaging (EPI) transverse images (TR $4000 \mathrm{~ms}$, TE $30 \mathrm{~ms}$, Flip Angle 90, FOV $208 \mathrm{~mm}$, matrix $64 \times 64$, SENSE Factor 2, 36 slices, $3 \mathrm{~mm}$ with $0 \mathrm{~mm}$ gap, transverse slice AC-PC orientation, with a voxel size of $3.25 \mathrm{~mm} \times 3.25 \mathrm{~mm} \times 3 \mathrm{~mm}$ ) were obtained covering the entire brain.

\section{Functional MRI Analyses}

\section{Whole Brain Meditation Minus Control and Control Minus Meditation Contrasts}

Functional imaging analysis was conducted using Statistical Parametric Mapping software (SPM 2, Wellcome Department of Cognitive Neurology, London, UK, using Matlab version 6.5.2). Functional imaging data of individual subjects were realigned, unwarped, spatially normalized to the EPI template in SPM2 and spatially smoothed using a SPM2 batched script (C. Rorden) to generate preprocessed images for statistical analysis. To generate contrast maps, the block design was convolved with a hemodynamic response function. Effects at each voxel were estimated using the general linear model within SPM2 at the first statistical level. The motionrecorded parameters created during the realign process were used as six user-specified regressors. A high pass filter of double the length of the longest trial $(720 \mathrm{~s})$ was 
used to remove low-frequency noise without impacting the signal of interest. Individual t-maps were generated by defining the following contrasts: Meditation-Control (respectively, $12 \mathrm{~min} .-6 \mathrm{~min}$.) and Control-Meditation (respectively $6 \mathrm{~min} .-12 \mathrm{~min}$.). These individual t-maps were used to generate group t-maps at the second level in a random effects model analysis (14). Cluster analyses were performed at a corrected threshold of $P<0.05$ (false discovery rate, FDR) for each group map with a spatial extent threshold of 20 voxels to account for multiple comparisons (15).

\section{Time Curves of Late Minus Early Meditation and Long/Shorter Term Practice}

SPM2, Analysis of Function Neuroimaging (AFNI) 2.56b, MRICro 1.37, and Excel 2000 were used to plot and graph time courses for region of interests (ROIs) (DLPFC and ACC) for further examination of regional brain activity over the course of meditation and control conditions. This novel method for extracting BOLD fMRI time courses has been reported elsewhere $(16,17)$. The individual t-maps and group maps were derived using the identical methods mentioned above. In order to distinguish possible late versus early changes in brain activity during meditation, the group t-map used to define the ROIs was of late Meditation-early Meditation using the last 2 min minus the first 2 min of each meditation block. The cluster analysis threshold of $P<0.001$ with a spatial extent of uncorrected $P<0.05$ was applied as the intent was to select for activation of ROIs during late minus early meditation. The subsequent surviving clusters were viewed in MRICro (18) to assess which clusters corresponded to the a priori predicted ROIs (i.e. DLPFC and ACC). AFNI (19) was used to plot BOLD signal time courses from activated voxels within the ROIs and was averaged for each individual. The BOLD signal values for each subject were combined in Excel 2000 to graph percent signal change (PSC) over the meditation and control conditions. Three group time courses were plotted: entire group; subgroup with $>10$ years' practice; and subgroup with $<10$ years' practice. This latter subgrouping was determined by post hoc analysis looking for a bimodal distribution in years of meditation practice.

Hierarchical linear modeling (HLM) was used to assess statistical significance of the effects of group membership (high experience versus low experience), condition (meditation versus control) and session (Run1 versus Run2) on individuals' patterns of ACC and DLPFC activation. HLM has been shown to appropriately handle nested models with serially dependent data points $(20,21)$. It allows for modeling of variables at the individual subject level (level 1; e.g. each subject's slope of cortical activation over time), at the broader organizational level to which each individual belongs (level 2; e.g. group) and/or within-subject experimental conditions (e.g. control versus meditation). The estimation method of the models was restricted maximum likelihood (REML) and the covariance structure was 'Unstructured'. Intercepts at the individual subject level were entered into the model as random effects.

\section{Results}

\section{Subject Demographics}

Thirteen right-handed subjects (8 women), aged 27-59 years (average age $43 \pm 9$ years), participated. Each participant meditated regularly for at least 4 years (mean $=11.6 \pm 9.4$ years). Daily meditation practice varied from $20 \mathrm{~min}$ a day to over $1 \mathrm{~h}$ a day, averaging approximately $30 \mathrm{~min}$ a day. All subjects practiced meditation but engaged in other professions. Six subjects were Tibetan Buddhists, three were Zen Buddhists, three were OM Yoga practitioners and one subject had had training in Tibetan and Zen Buddhism. Within the scanner, all subjects reported entering a meditative state that varied over time. The five subjects with $>10$ years of meditative practice qualitatively reported a similar experiential progression of focusing attention on breathing and a relative quiescence of other contents of the mind consistent with their regular practice. The other eight subjects with less practice reported more varied experiences. Five acknowledged better concentration at the beginning, one in the middle and two during the final minutes of the meditation. Generally their meditative states, however, were not as in-depth or as focused as their routinely practiced meditative sessions. Most subjects reported minimal distraction from the fMRI sounds, and their distraction decreased over time.

\section{Structural MRI Results}

No significant lesions were noted on T1-weighted structural scans or T2*-weighted EPI images. One subject did not have a T1-weighted structural scan due to equipment failure.

\section{Functional MRI Results}

\section{Meditation minus control and control minus meditation}

No regions were significantly increased in the meditation minus control comparisons using the a priori defined threshold of FDR, statistical threshold of $P<0.05$ and extent threshold of 20 voxels. Control minus meditation revealed activation in the bi-occipital [Brodmann areas (BA) 17, 18, 19], bi-cerebellar, bi-occipitotemporal (BA 37), left temporal (BA 20, 21, 22), left angular parietal (BA 22, 39) and left inferior orbital frontal (BA 47) cortex. 


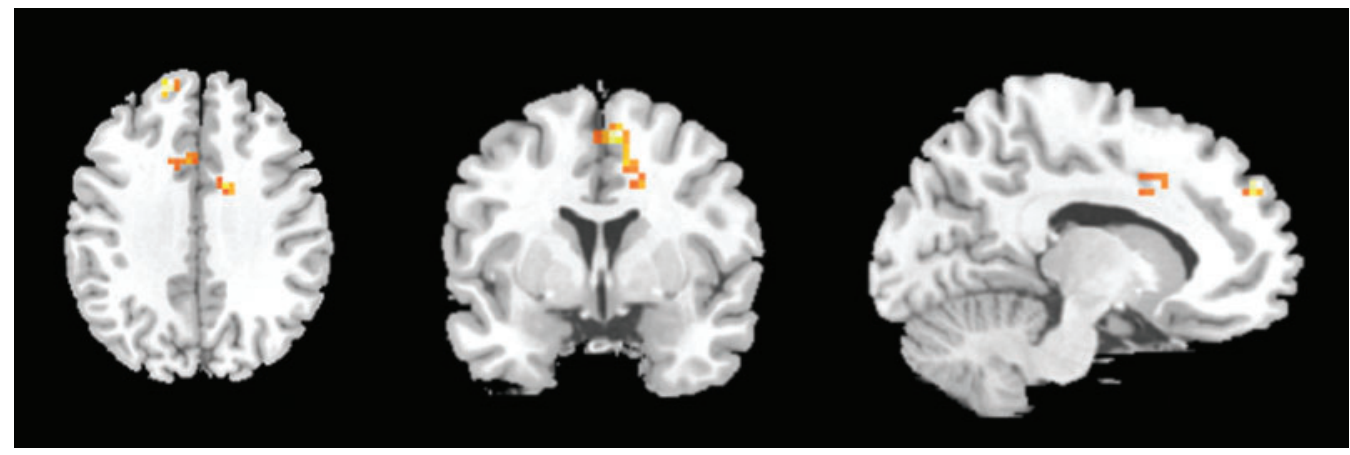

Figure 1. ROIs in late minus early meditation. Brain activation in left dorsal lateral frontal cortex (BA 9) and ACC (BA 24, 32) $(P<0.001$, extent threshold $P<0.05$, no FDR) in a contrast map of all subjects in late minus early meditation.

Time curves of late minus early meditation and long/shorter term practice

A contrast map for all subjects in late minus early meditation (last 2 min minus first 2 min) revealed brain activation in left dorsal lateral frontal cortex (BA 9) and ACC (BA 24, 32) $(P<0.001$, extent threshold $P<0.05$, no FDR). Further analysis was employed only for these two clusters.

\section{ACC BOLD Signal Time Course}

For the model estimating ACC activation, the covariance parameter estimates were different from 0 at both the subject level $(2787.21, Z=2.32, P<0.01)$ and residual (2038.25, $Z=83.39, P<0.0001)$. The intraclass correlation coefficient was 0.58 suggesting that there is clustering of activation patterns within subject and that ordinary least squares analysis of these data might yield misleading results (22). A significant main effect was detected for session (Run1, Run2) $[F(1,11)=98.43, P<0.0001]$ and the group (meditation $<10$ years, meditation $>10$ years) $\times$ session interaction was also significant $[F(1,11)=$ 191.91, $P<0.0001]$. Thus, HLM was used to perform post-hoc models separately for each session.

There was an increased mean ACC activation during meditation in comparison with control tasks for both sessions (session $1 T=4.96, P<0.001$; session 2 $T=36.33, P<0.0001)$. In the less-experienced group, mean ACC activity was lower during meditation than during the control condition (session $1 T=7.65$, $P<0.0001$; session $2 T=13.13, P<0.0001)$. However, ACC activation during meditation increased over time relative to control (session $1 T=2.36, \mathrm{P}<0.02$; session 2 $T=9.41, P<0.0001)$. In the more experienced group, the mean ACC activity was higher during meditation than the control condition for both sessions (session 1 $T=5.59, P<0.01$; session $2 T=7.67, P<0.01)$. ACC activation increased over time during meditation relative to the control condition in session $1 \quad(T=5.37$, $P<0.0001)$ but decreased over time in session 2 $(T=2.03, P<0.05)$.

\section{DLPFC BOLD Signal Time Course}

For the model estimating DLPFC activation, the covariance parameter estimates were different from 0 at both the subject level (6788.41, $Z=2.34, P<0.01$ ) and residual (2112.71, $Z=83.39, P<0.0001)$. The intraclass correlation coefficient was 0.76 , again suggesting that there is clustering of activation patterns within subject and that ordinary least squares analysis of these data might yield misleading results (22). Much like the results from the HLM of ACC activity, a significant main effects was observed for session (Run1, Run2) $[F(1,11)=135.59$, $P<0.0001]$ and the group (meditation $<10$ years, meditation $>10$ years $) \times$ session interaction $[F(1,11)=$ 194.68, $P<0.0001]$ on DLPFC activity.

Overall, there was increased DLPFC activation during meditation in comparison with the control condition during session 2 (session $1 T=1.10$, NS, session $2 T=6.61$, $P<0.0001)$. In the less-experienced group, there was more DLPFC activation during meditation than the control condition in session 1 (session $1 T=10.67, P<0.0001$; session $2 T=1.02$, NS). However, DLPFC activity decreased over time during meditation relative to the control condition in both sessions (session $1 T=10.16$, $P<0.0001$; session $2 T=3.66, P<0.001)$. In the more experienced group, there was more DLPFC activity during meditation than the control condition in session 2 (session 1 $T=0.94$, NS; session $2 T=3.15, P<0.01)$. Furthermore, DLPFC activity increased over time during meditation relative to the control condition during session 1 (session 1 $T=.54, P<0.001$; session $2 T=10, \mathrm{~ns}$ ).

\section{Discussion}

Using a general linear model whole brain analysis, we failed to confirm our hypotheses of increased activity in DLPFC and ACC during meditation. However, our hypothesis was supported by using a region-specific and time-sensitive methodology of plotted time courses.

To our knowledge, this is the first fMRI meditation work that plots time courses of brain regions associated with attention over $12 \mathrm{~min}$ in subjects from different meditative 
A. Entire Group $(\mathrm{n}=13)$

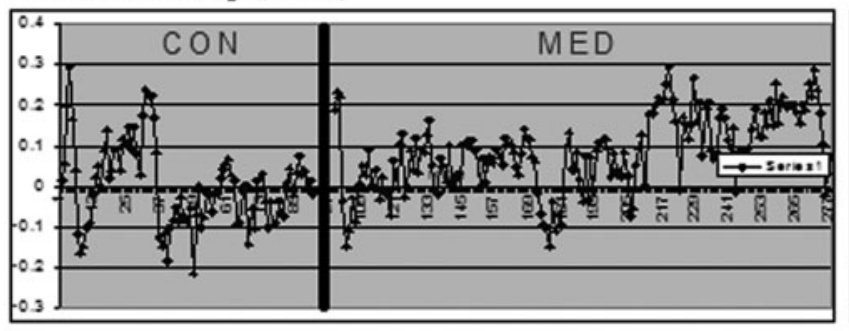

B. Group of $<10$ years meditation experience $(n=8)$

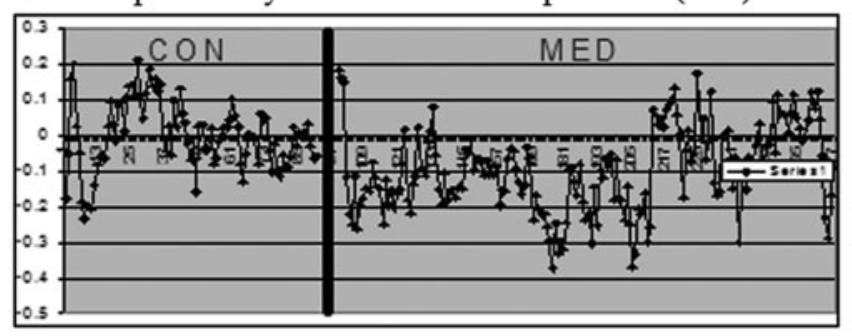

C. Group of $>10$ years meditation experience $(n=5)$

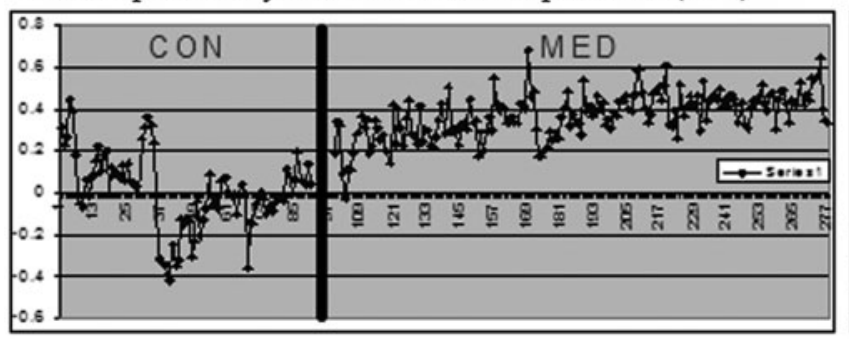

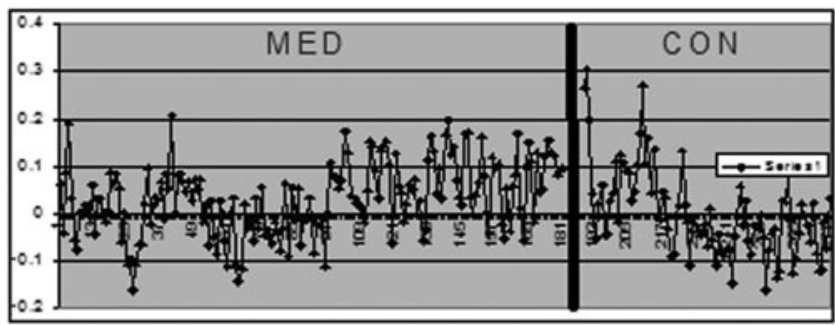
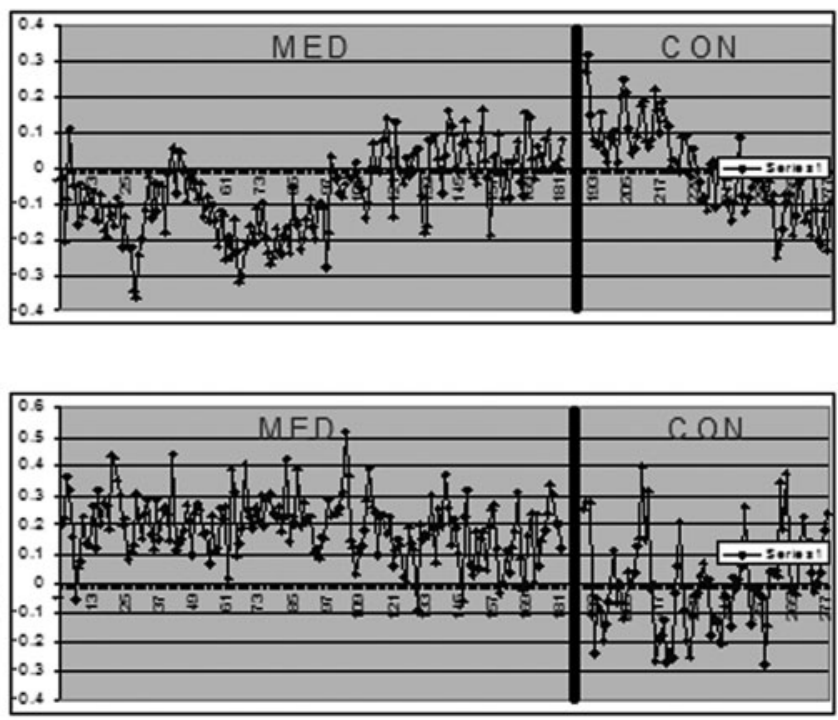

Figure 2. BOLD time curves of the ACC. Patterns of BOLD response time curves of ACC for entire group (A), 'subjects practiced meditation for $<10$ years' group $(\mathbf{B})$ and 'subjects practiced meditation $>10$ years' group $(\mathbf{C})$. The two time courses represent the two fMRI runs for each group, which are labeled CON for control and MED for meditation tasks. The horizontal, straight line represents average percent BOLD signal of ACC activity during averaged control. The horizontal, serpentine line represents the mean percent BOLD signal change of ACC.

traditions. The entire group-averaged time courses revealed significant increases in percent BOLD responses in ACC and DLPFC during the meditation tasks over control tasks (trend for session 1 DLPFC). There were significantly different ACC and DLPFC activation patterns over time between long- and short-term practitioners during meditation. These results may involve possible dissociable roles of ACC and DLPFC in cognitive attentional control. One analysis suggests meditation may enhance specific subcomponents of attention such as conflict monitoring or performance (23). DLPFC may involve attentional maintenance while ACC monitors performance (24). Speculatively, the shorter term practitioners' ( $<10$ years) attentional maintenance decreased over time, corresponding with the decreased DLPFC over the time of meditation. This may have led to increased error monitoring of wandering attention over time, which correlated with increased ACC activity over time. The long-term practitioners ( $>10$ years) may have maintained attention on the meditation task over time better than shorter term practitioners, which correlated with increased mean DLPFC activity over time. Long-term practitioners had higher mean ACC activity in comparison with control tasks, unlike the shorter term practitioners. Long-term practitioners possibly had better error monitoring of wandering attention than shorter term practitioners. The decreasing ACC activity over time in the second session for long-term practitioners may correlate with a decreased need for error monitoring of wandering attention because of the high attentional maintenance related to DLPFC activity. This work suggests there may be attentional state differences between subjects with long- and short-term meditation practice. However, because of the correlative nature of these measures, firm causal conclusions are not warranted. Interestingly, these results are consistent with a recent study describing more active DLPFC activity and decreased ACC activity in expert meditators in comparison with novices during meditation over shorter time epochs (25). Further longitudinal work is needed to assess attentional changes associated with meditation practice.

Limitations of this study include the small sample size, lack of statistical significance with whole brain analysis using conservative a priori defined thresholds, subject variability in meditation practice and duration, no prior experience meditating in the MRI scanner, a lack of psychophysiological measures during the scan (e.g. heart rate) 
A. Entire Group $(\mathrm{n}=13)$
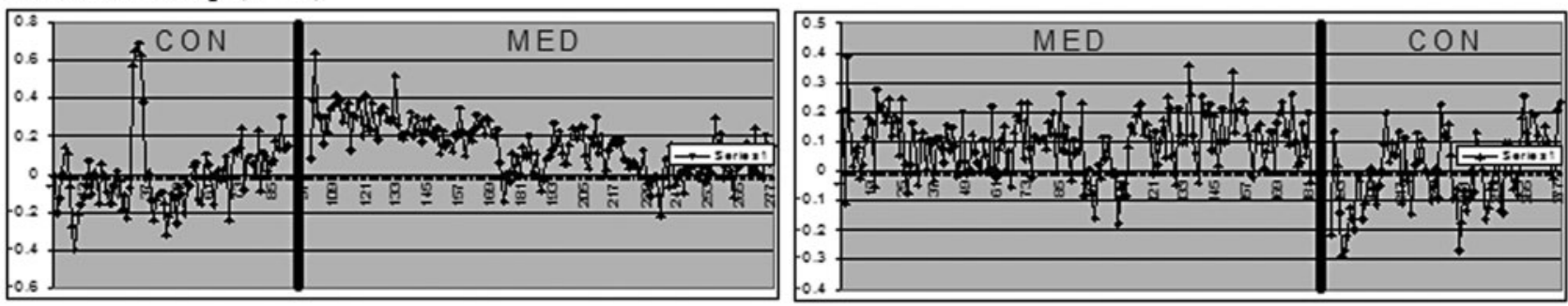

B. Group of $<10$ years meditation experience $(\mathrm{n}=8)$
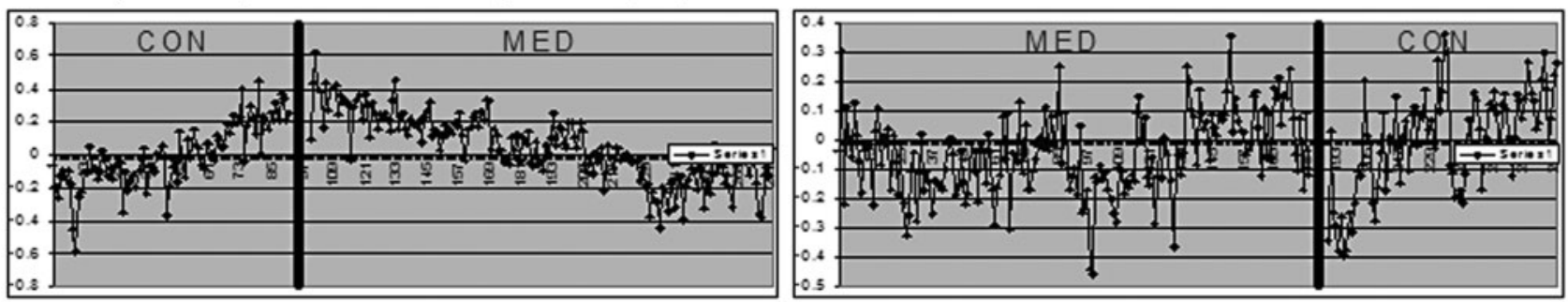

C. Group of $>10$ years meditation experience $(n=5)$
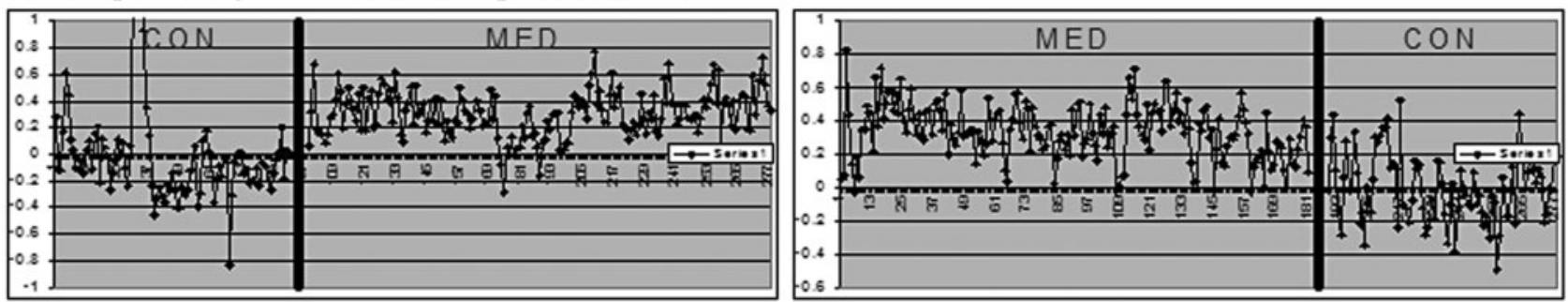

Figure 3. BOLD time curves of the DLPFC. Patterns of BOLD response time curves of DLPFC for entire group (A), for 'subjects practiced meditation < 10 years' group (B) and 'subjects practiced meditation $>10$ years' group (C). The two time courses represent the two fMRI runs for each group, which are labeled CON for control and MED for meditation tasks. The horizontal, straight line represents average percent BOLD signal of DLPFC activity during averaged control. The horizontal, serpentine line represents the mean percent BOLD signal change of DLPFC.

and meditation/control condition interpretations. Individual differences of practice duration and subtle discrepancies in breath awareness meditation may have confounded whole brain analyses, while region-specific time-sensitive analyses still revealed differences in longand short-term meditators. All subjects had meditation practice with eyes partially open, but few had meditated while lying down, and no one had meditated before, in a MRI scanner. However, all subjects were instructed to meditate lying down with eyes open while listening to an audio tape of fMRI sounds until comfortably entering a meditative state with breath awareness for approximately 2 weeks before scanning. The absence of psycho-physiological measures limits validation that all subjects had engaged in a similar meditative physiological response that could then be correlated with functional MRI findings. The increased occipital brain activity in control minus meditation contrast was consistent with a more visually active control task. The increased inferior parietal activity could represent relative activation during control versus inhibition during meditation. Inferior parietal functionality may include encoding for body schema and part of the attention network $(26,27)$.
Further work using the a priori defined HLM analysis method is required to confirm these neuroanatomic functional correlates of meditation.

In conclusion, using BOLD fMRI signal time courses, we found that brain activity in regions associated with attention vary over the time of a meditation session and differ between long- and short-term practitioners.

\section{Acknowledgements}

Grant support was received from a grant in kind from the Center for Advanced Imaging Research (CAIR) and the University Research Committee at MUSC. Subject participation support was received from the Charleston Tibetan Society and the Charleston Zen Center.

Conflict of Interest: Jeffery Borckardt currently conducts research funded by NINDS at NIH and by Cyberonics, Inc.

\section{References}

1. Austin J. Zen-Brain Reflections. Cambridge: The MIT Press, 2006.

2. Cahn BR, Polich J. Meditation states and traits: EEG, ERP, and neuroimaging studies. Psychol Bull 2006;132:180-211. 
3. Casey BJ, Thomas KM, Welsh TF, Badgaiyan RD, Eccard $\mathrm{CH}$, Jennings JR, et al. Dissociation of response conflict, attentional selection, and expectancy with functional magnetic resonance imaging. Proc Natl Acad Sci USA 2000;97:8728-33.

4. Duncan J, Owen AM. Common regions of the human frontal lobe recruited by diverse cognitive demands. Trends Neurosci 2000;23:475-83.

5. Smith EE, Jonides J. Storage and executive processes in the frontal lobes. Science 1999;283:1657-61.

6. Kondo H, Osaka N, Osaka M. Cooperation of the anterior cingulate cortex and dorsolateral prefrontal cortex for attention shifting. Neuroimage 2004;23:670-9.

7. Carter CS, Braver TS, Barch DM, Botvinick MM, Noll D, Cohen JD. Anterior cingulate cortex, error detection, and the online monitoring of performance. Science 1998;280:747-9.

8. Rowe JB, Toni I, Josephs O, Frackowiak RS, Passingham RE. The prefrontal cortex: response selection or maintenance within working memory? Science 2000;288:1656-60.

9. Bunge SA, Ochsner KN, Desmond JE, Glover GH, Gabrieli JD. Prefrontal regions involved in keeping information in and out of mind. Brain 2001;124:2074-86.

10. Lazar SW, Kerr CE, Wasserman RH, Gray JR, Greve DN Treadway MT, et al. Meditation experience is associated with increased cortical thickness. Neuroreport 2005;16:1893-7.

11. Carter OL, Presti DE, Callistemon C, Ungerer Y, Liu GB, Pettigrew JD. Meditation alters perceptual rivalry in Tibetan Buddhist monks. Curr Biol 2005;15:R412-13.

12. Pagnoni G, Cekic M. Age effects on gray matter volume and attentional performance in Zen meditation. Neurobiol Aging 2007;24:24.

13. Hankey A. Studies of advanced stages of meditation in the tibetan buddhist and vedic traditions. I: a comparison of general changes. Evid Based Complement Alternat Med 2006:3:513-21.

14. Reiman EM, Lane RD, Ahern GL, Schwartz GE, Davidson RJ, Friston KJ, et al. Neuroanatomical correlates of externally and internally generated human emotion. Am $J$ Psychiatry 1997:154:918-25.
15. Friston KJ, Worsley KJ, Frackowiak RJ, Mazziotta JC, Evans AC. Assessing the significance of focal activations using their spatial extent. Hum Brain Mapp 1994;1:214-220.

16. $\mathrm{Mu} \mathrm{Q}$, Mishory A, Johnson KA, Nahas Z, Kozel FA, Yamanaka K, et al. Decreased brain activation during a working memory task at rested baseline is associated with vulnerability to sleep deprivation. Sleep 2005;28:433-46.

17. Mu Q, Nahas Z, Johnson KA, Yamanaka K, Mishory A, Koola J, et al. Decreased cortical response to verbal working memory following sleep deprivation. Sleep 2005;28:55-67.

18. Rorden C. MRICro. 2003.

19. Cox RW. AFNI: software for analysis and visualization of functional magnetic resonance neuroimages. Comput Biomed Res 1996;29:162-73.

20. Bryk AsaR, S.W. Application of hierachical linear models to assessing change. Psychol Bull 1987;101:147-58.

21. Bryk AS, Raudenbush SW. Hierarchical linear models: Applications and data analysis methods. Newbury Park, CA: Sage Publications, 1992.

22. Singer JD. Using SAS PROC MIXED to fit multilevel models, hiearchical models and individual growth curves. 1998.

23. Jha AP, Krompinger J, Baime MJ. Mindfulness training modifies subsystems of attention. Cogn Affect Behav Neurosci 2007;7:109-19.

24. MacDonald AW 3rd, Cohen JD, Stenger VA, Carter CS Dissociating the role of the dorsolateral prefrontal and anterior cingulate cortex in cognitive control. Science 2000;288:1835-38.

25. Brefczynski-Lewis JA, Lutz A, Schaefer HS, Levinson DB, Davidson RJ. Neural correlates of attentional expertise in longterm meditation practitioners. Proc Natl Acad Sci USA 2007;104:11483-8.

26. Chaminade T, Meltzoff AN, Decety J. An fMRI study of imitation: action representation and body schema. Neuropsychologia 2005:43:115-27.

27. Fan J, McCandliss BD, Fossella J, Flombaum JI, Posner MI. The activation of attentional networks. Neuroimage 2005;26:471-9.

Received May 18, 2007; accepted September 12, 2007 


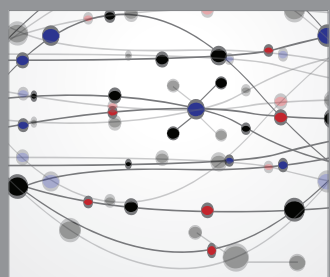

The Scientific World Journal
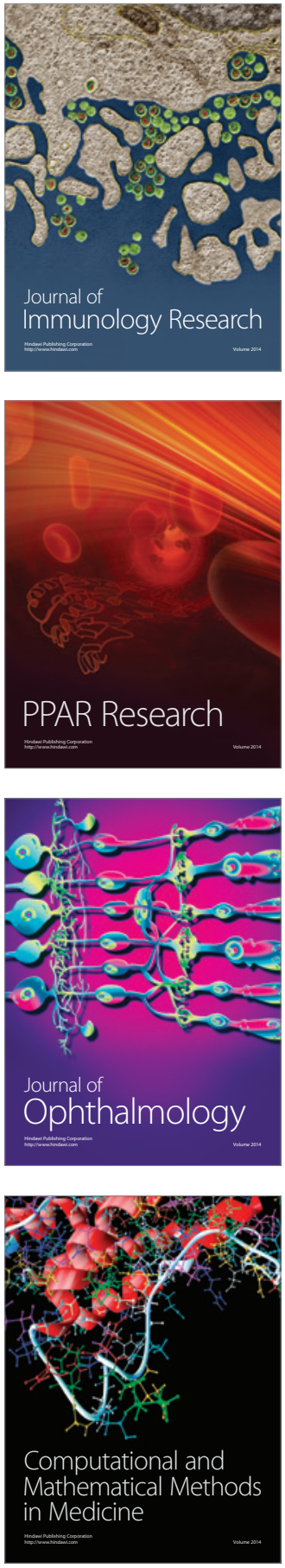

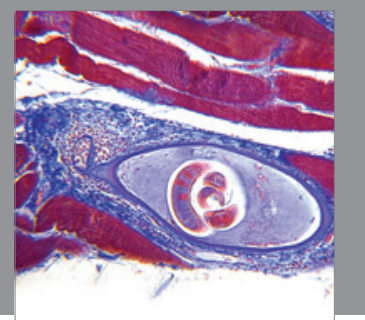

Gastroenterology

Research and Practice
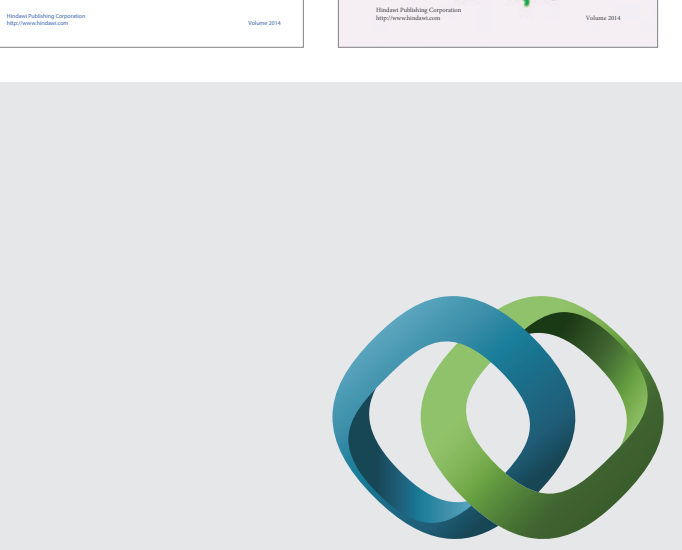

\section{Hindawi}

Submit your manuscripts at

http://www.hindawi.com
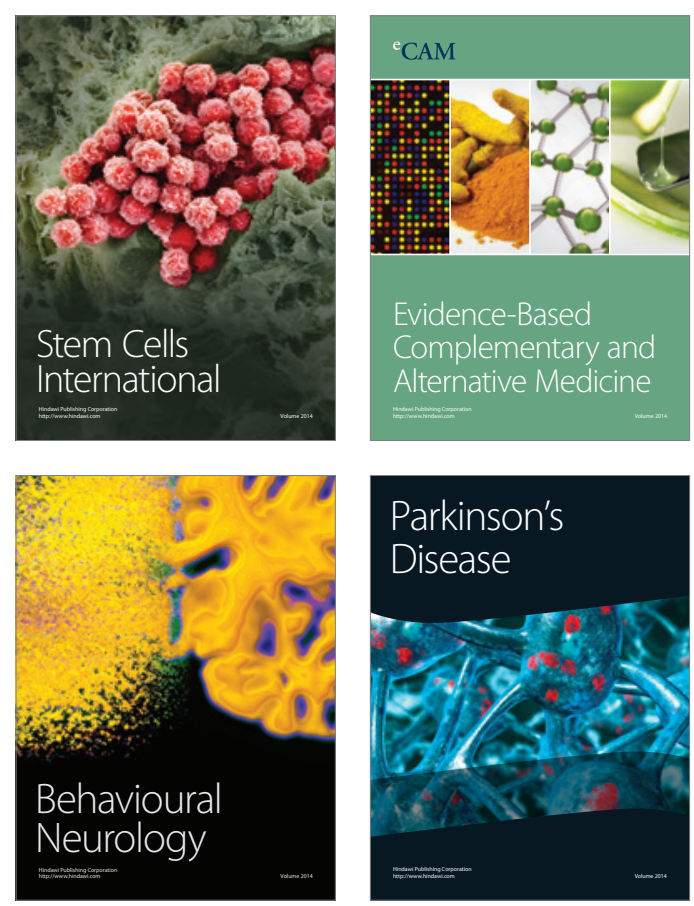

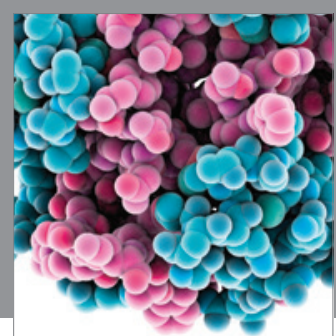

Journal of
Diabetes Research

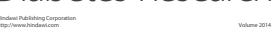

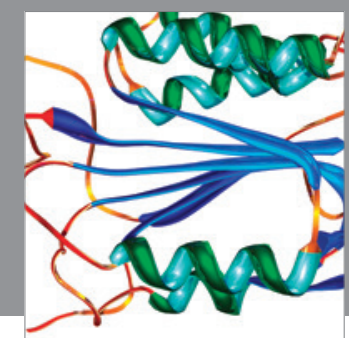

Disease Markers
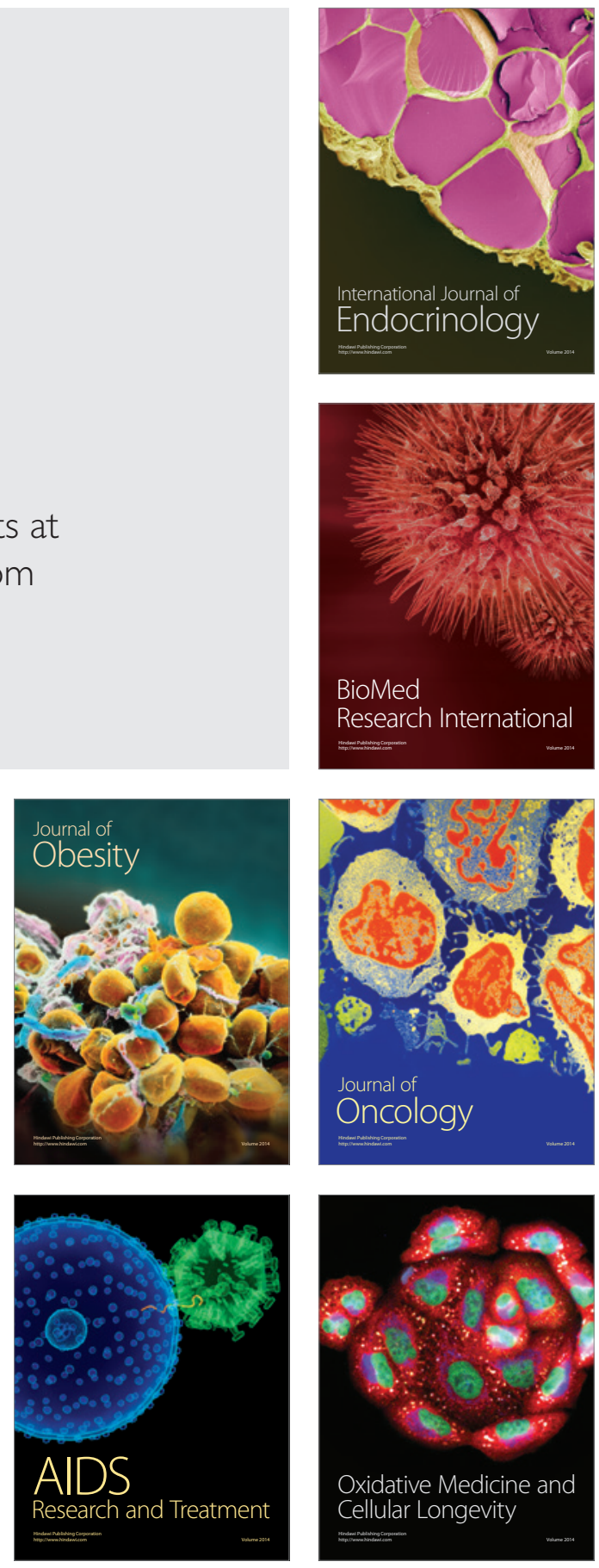